\title{
THE TRIGGER OF THE ALICE DIMUON ARM: ARCHITECTURE AND DETECTORS
}

\author{
R. Arnaldi d , A. Baldit ${ }^{\text {b }, ~ V . ~ B a r r e t ~}{ }^{\text {b }}$, N. Bastid ${ }^{\text {b }}$, G. Blanchard ${ }^{\text {b}}$, E. Chiavassa ${ }^{\text {d, }}$, \\ P. Cortese ${ }^{d}$, P. Crochet ${ }^{b}$, G. Dellacasa, ${ }^{a}$, N. De Marco ${ }^{d}$, P. Dupieux, ${ }^{b}$, \\ B. Espagnon ${ }^{\mathrm{b}}$, J. Fargeix ${ }^{\mathrm{b}}$, M. Gallio ${ }^{\mathrm{d}}$, L. Lamoine ${ }^{\mathrm{b}}$, L. Luquin, ${ }^{\mathrm{c}}$, F. Manso ${ }^{\mathrm{b}}$, \\ V. Metivier ${ }^{c}$, A. Musso ${ }^{\text {d }}$, A. Piccotti ${ }^{\text {d }}$, A. Rahmani ${ }^{\text {c }}$, V. Ramillen ${ }^{\text {b }}$, L. Royer ${ }^{\text {b }}$, \\ O. Roig ${ }^{\text {b }}$, E. Scalas ${ }^{\text {a }}$, E. Scomparin ${ }^{d}$, E. Vercellin ${ }^{d}$
}

For the ALICE collaboration

aINFN Torino and Universita' del Piemonte Orientale "Amedeo Avogadro", Alessandria ${ }^{b}$ Laboratoire de Phisyque Corpuscolaire de Clermont-Fd, IN2P3-CNRS-Universite' Blaise Plascal, Clermont Ferrand

${ }^{\mathrm{c}}$ Laboratoire de Physique Subatomic et des Technologies Associees, Nantes

${ }^{\mathrm{d}}$ INFN Torino and Dipartimento di Fisica sperimentale dell'Universita' di Torino

The trigger system of the ALICE dimuon arm is based on Resistive Plate Chambers (RPC). Besides a short description of the trigger system, the test results of a RPC prototype with electrodes made of low resistivity bakelite $\left(\simeq 3 \cdot 10^{9} \Omega \cdot \mathrm{cm}\right)$ are presented. Rate capability, time resolution and cluster size have been measured for the RPC operated both in streamer and in avalanche mode. Although the rate capability is obviously higher in avalanche mode (few $\mathrm{kHz} / \mathrm{cm}^{2}$ ), remarkable results have been achieved even in streamer mode (several hundreds of $\mathrm{Hz} / \mathrm{cm}^{2}$ ).

\section{INTRODUCTION}

The production of heavy quarkonia ( $\mathrm{J} / \Psi$ and $\Upsilon$ families) is expected to be sensitive to QGP formation and will be studied by the muon arm of the ALICE experiment at the LHC. Since the transverse momentum of background muons from $\pi$ and K decay is smaller than the one of muons emitted in quarkonia decays, a first selection of the events is done by triggering on high $p_{t}$ muon pairs.

The trigger detectors of the ALICE muon spectrometer will be Resistive Plate Chambers (RPC) [1,2] which are simple devices with performances well satisfing trigger purposes (fast response and good time resolution); futhermore they are industrially produced and suitable to cover large areas.

RPC tests have been performed in order to optimize the detector's performances for the ALICE experimental conditions. 


\section{THE ALICE DIMUON TRIGGER}

The experimental layout of the ALICE dimuon spectrometer is thorougly described in ref. [3]. The trigger system is made up of 2 trigger stations placed at the end of the spectrometer, respectively at $16 \mathrm{~m}$ and $17 \mathrm{~m}$ from the interaction point which cover an area of about $6 \times 6 \mathrm{~m}^{2}$. Each trigger station is made of 2 planes of 18 single gap RPCs read-out on both sides by means of strips in the two orthogonal directions. In the bending plane the strip widths are 1-2-4 cm, going from the inner to the outer part of the trigger stations, while in the non-bending plane, only two different strip widths are used: 2 and $4 \mathrm{~cm}$. With this configuration there are about 20000 read-out channels.

The informations from the trigger chambers are treated by a programmable trigger electronics in order to perform the cut on the single muon $p_{t}$ [4]. Two $p_{t}$ cuts are foreseen: $p_{t}>1 \mathrm{GeV} / \mathrm{c}$ for the $\mathrm{J} / \Psi$, and $p_{t}>2 \mathrm{GeV} / \mathrm{c}$ for the $\Upsilon$. The trigger performs a selection of events with at least 2 tracks of opposite sign which satisfy the $p_{t}$ cut and delivers a signal after $\simeq 600 \mathrm{~ns}$.

\section{RPC FOR THE ALICE DIMUON ARM}

RPC can be operated in streamer or in avalanche mode, the first one imposes severe constraints on the rate capability of the detector because of the large charge released that causes a local reduction of the voltage between the electrodes. As the RPCs of the ALICE muon arm are expected to be fired at rates lower than $50 \mathrm{~Hz} / \mathrm{cm}^{2}$ the possibility to operate RPC in streamer mode has been deeply investigated optimizing the two main parameters: the gas mixture and the electrode resistivity. The former affects the amount of charge released in the streamer and the quality of the signals, the latter determines the recovery time of the detector.

Besides a rate capability of $\simeq 100 \mathrm{~Hz} / \mathrm{cm}^{2}$, which take into account a safety factor with respect to the simulations, a time resolution sufficiently good $(\sim 3 \mathrm{~ns})$ to strobe the RPC signals within a $20 \mathrm{~ns}$ wide gate is required in order to reduce the background hits. As well as a small cluster size is required in order to minimize the occupancy of the read-out strips.

\subsection{Beam test}

A RPC prototype of small dimensions $\left(50 \times 50 \mathrm{~cm}^{2}\right)$ operating both in streamer and in avalanche mode has been tested at the CERN SPS. The $2 \mathrm{~mm}$ thick electrodes are made of low resistivity bakelite $\left(\rho \simeq 3 \cdot 10^{9} \Omega \cdot \mathrm{cm}\right)$, between them there is a $2 \mathrm{~mm}$ thick gas gap. The gas mixture for streamer mode have been optimized with cosmic rays tests [5] and was: $49 \% \mathrm{Ar}+40 \% \mathrm{C}_{2} \mathrm{H}_{2} \mathrm{~F}_{4}+7 \% \mathrm{C}_{4} \mathrm{H}_{10}+4 \% \mathrm{SF}_{6}$; while for avalanche mode was $95 \% \mathrm{C}_{2} \mathrm{H}_{2} \mathrm{~F}_{4}+3 \% \mathrm{C}_{4} \mathrm{H}_{10}+2 \% \mathrm{SF}_{6}[6]$.

Efficiency, time resolution and cluster size have been measured for local fluxes $\left(2 \times 2 \mathrm{~cm}^{2}\right)$ in the center of the chamber.

\subsection{Efficiency and rate capability}

The efficiency has been measured as a function of H.V.: the efficiency plateau starts at $8.8 \mathrm{kV}$ for the streamer and at $9.9 \mathrm{kV}$ for the avalanche mode. The efficiency evolution 


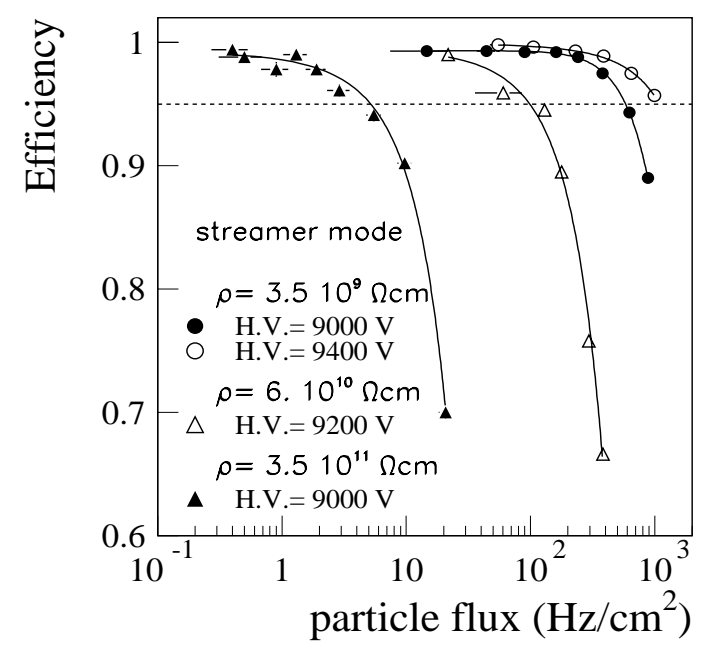

Figure 1. Efficiency as a function of the incident flux for RPC operating in streamer mode.

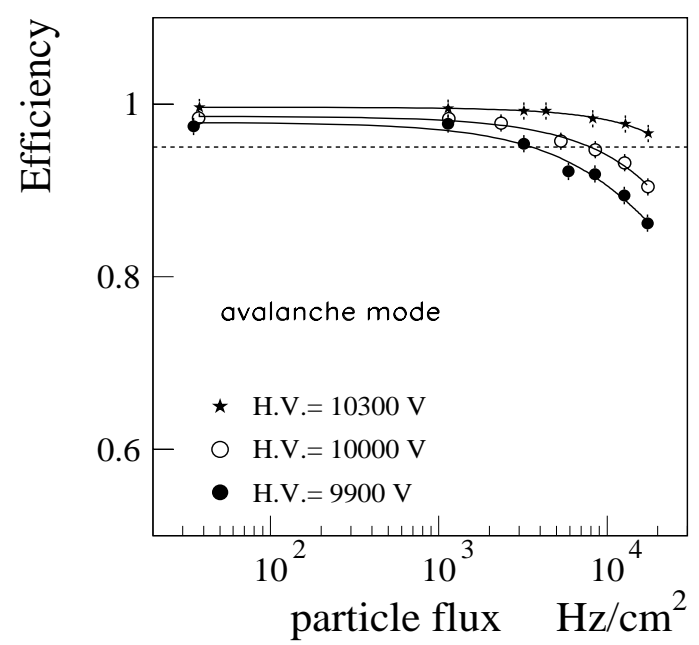

Figure 2. Efficiency as a function of the incident flux for the low resistivity RPC in avalanche mode.

as a function of flux is summarized in fig. 1 and 2. In fig. 1 is shown the efficiency for the streamer mode at two different values of the high voltage. It is still better than $95 \%$ for fluxes around $500 \mathrm{~Hz} / \mathrm{cm}^{2}$ and $1 \mathrm{kHz} / \mathrm{cm}^{2}$ at $9 \mathrm{kV}$ and $9.4 \mathrm{kV}$ respectively. To emphasize the straightforward dependence of the rate capability on the resistivity of the electrodes, we have also plotted in fig. 2 the efficiencies of two RPCs made with bakelite of higher resistivity, respectively of about $3.5 \cdot 10^{11} \Omega \cdot \mathrm{cm}$ and $6 \cdot 10^{10} \Omega \cdot \mathrm{cm}[5]$. The efficiency obtained when the low resistivity RPC is operated in avalanche mode is visible in fig. 2 . As it can be seen, also in this case, better rate capabilities are achieved by increasing the high voltage: at $10.3 \mathrm{kV}$ the efficiency is still better than $95 \%$ at $10 \mathrm{kHz} / \mathrm{cm}^{2}$.

\subsection{Time resolution}

The time performance of the RPC operated in streamer mode is summarized in fig. 3, where, for H.V. $=9.4 \mathrm{kV}$, the r.m.s. of the TDC distribution are plotted as a function of the incident flux. Fig. 3 shows that the r.m.s. is $\sim 1$ ns below $100 \mathrm{~Hz} / \mathrm{cm}^{2}$ and then it increases up to $\sim 4 \mathrm{~ns}$ for incident fluxes of the order of $1 \mathrm{kHz} / \mathrm{cm}^{2}$. In fact when the flux increases the width of the TDC peak as well as its position are basically unchanged, but a tail appears on the right side of the peak. However, even at $1 \mathrm{kHz} / \mathrm{cm}^{2}$, at $9.4 \mathrm{kV}$ more than $98 \%$ of the events are included in a 20 ns window.

For the avalanche mode, the TDC distribution measured at $10 \mathrm{kV}$ is nearly gaussian, with a r.m.s. of $0.9 \mathrm{~ns}$ and it does not show a significant dependence from high voltage and particle flux.

\subsection{Cluster size}

In fig. 4 are compared the cluster size distributions between the streamer mode and the avalanche mode for RPC equipped with $2 \mathrm{~cm}$ wide strips. For the streamer mode the mean value of the cluster size is 1.12 and the fraction of events where more than three adjacent strips are fired is smaller than $10^{-3}$. The cluster size has been measured also for 


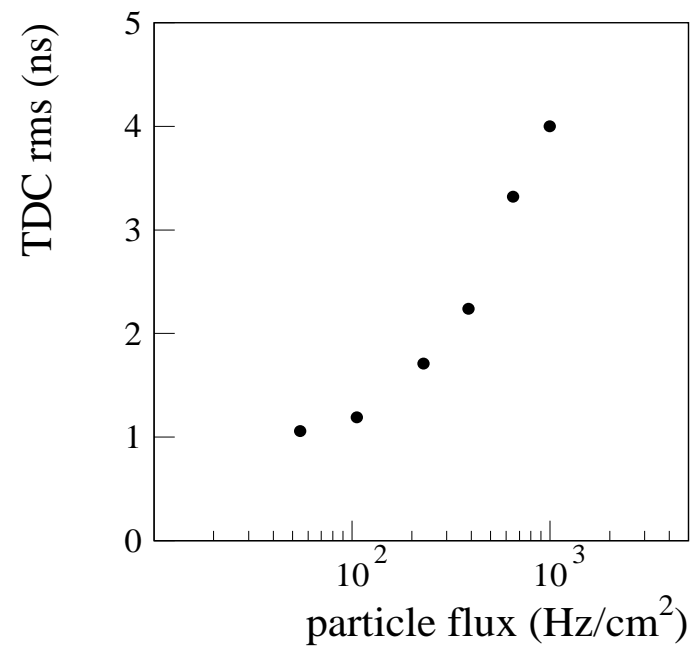

Figure 3. Root mean square of the time distribution as a function of the incident flux. The data are for the streamer mode.

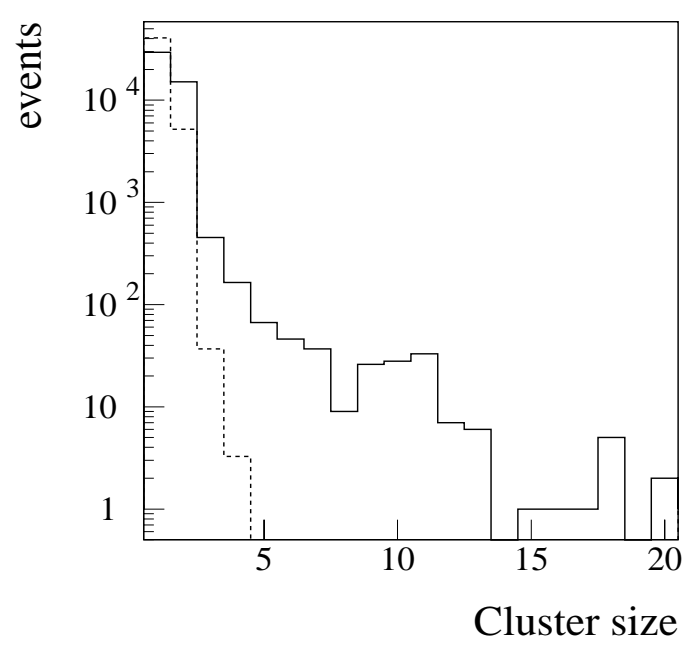

Figure 4. Cluster size distribution in streamer mode (dashed line) and in avalanche mode (full line) for $2 \mathrm{~cm}$ strips.

$1 \mathrm{~cm}$ wide strips, in this case the mean value is 1.46 . For the avalanche mode the cluster size has been measured only for $2 \mathrm{~cm}$ wide strips. The distribution is remarkably different: the mean value is higher (1.41) and there is a much larger fraction of events (about 1\%) with more than three adjacent fired strips.

\section{CONCLUSIONS}

The ALICE experiment at LHC will need a performant trigger to select high transverse momentum muon pairs emitted in the decay of heavy quarkonia $(\mathrm{J} / \Psi$ and $\Upsilon)$. To achieve this goal, there is need for low-cost detectors with high segmentation and good time resolution: requirements fulfilled by Resistive Plate Chambers.

A study of RPC with electrodes made of low resistivity bakelite $\left(\rho \simeq 3 \cdot 10^{9} \Omega \cdot \mathrm{cm}\right)$ was performed at the SPS in order to define the parameters of the chambers which will be used in the Alice dimuon trigger. Rate capability, cluster size and time resolution were measured for the RPCs operating in streamer mode and in avalanche mode.

The encouraging results obtained for operation in streamer mode indicate that it could represent a viable solution for the ALICE experiment.

\section{REFERENCES}

1. R. Santonico and R. Cardarelli, Nuc. Instr. and Meth. 187 (1981), 377

2. R. Cardarelli, V. Makeev and R. Santonico, Nuc. Instr. and Meth. A 382 (1996), 470

3. Addendum to the ALICE Technical Proposal, CERN/LHCC 96-32, (1996)

4. ALICE collaboration, Internal note/DIM, ALICE/98-18, (1998)

5. ALICE collaboration, Internal note/DIM, ALICE/98-16, (1998)

6. P. Camarri et al., Nuc. Instr. and Meth. A 414 (1998), 317 